ISSN 0103-9954

\title{
ANÁLISE DE ALGUMAS ALTERNATIVAS PARA OBTER O COEFICIENTE ANGULAR DA RETA NO MÉTODO DA ALTURA RELATIVA
}

\author{
ANALYSIS OF SOME ALTERNATIVES TO CHARACTERIZE THE TREE PROFILE OF EUCALYPT \\ USING ANALITICAL GEOMETRY
}

\author{
Valdir Carlos Lima de Andrade $^{1}$ Natalino Calegario ${ }^{2}$ José Roberto Soares Scolforo ${ }^{3}$ \\ RESUMO
}

O método da altura relativa permite caracterizar o perfil do tronco sem precisar do abate de árvoresamostra para cubagem. Emprega-se geometria analítica nas variáveis obtidas partindo de diâmetros tomados a 0,3 metros do terreno e em $h r=(h-2) / 2$, além de $d_{1,3}$ e $h$, o que viabiliza a medição destas, simultaneamente, com as demais variáveis mensuradas nas parcelas de inventário florestal. Neste trabalho, foram avaliadas algumas opções para caracterização do perfil do tronco empregando geometria analítica, comparando-se com o seu uso original conforme Andrade (2001). Concluiu-se que o uso de diâmetros relativos ao dap apresentaram melhores resultados para empregar a análise de regressão, a fim de se gerar coeficientes angulares da reta. Também, na expectativa de melhor acurácia, é mais adequado empregar o método da altura relativa utilizando todas as árvores delimitadas pelas parcelas do inventário.

Palavras-chave: análise de regressão; cubagem de árvores; método da altura relativa.

\section{ABSTRACT}

The relative height method allows the characterization of stem profile without meeding sample trees to upper diameter measurements. The analytical geometry is applied on the diameter taken on 0,3 meters and on $\mathrm{hr}=(\mathrm{h}-2) / 2$, as well as dbh and total height $(\mathrm{h})$, making possible the measurement of these variables, simultaneously, with others measured on the permanent sampling in forest inventory. This study evaluated some alternatives in characterizing the stem profile by using analytical geometry, comparing it to the original method according Andrade (2001). It was concluded that the use of relative diameters to dbh showed better results in regression analysis to generate straight line angular coefficient. Also, to obtain better accuracy, it is more adequate to implement the relative height method using all trees inside the inventory sample unit.

Keywords: regression analysis; stem analysis; relative height method.

\section{INTRODUÇÃO}

A cubagem de árvores é essencial para se caracterizar o comportamento do diâmetro ao longo do tronco bem como de seu volume, que pode ser obtido pelo emprego de diferentes fórmulas de cubagem, das quais, se destacam as fórmulas de Smalian, Huber e de Newton que, conforme relatos de Finger (1992), Machado e Figueiredo-Filho (2003) e Scolforo (2005), foram introduzidas no setor florestal entre o final do século XVIII e início do século XIX.

Os dados obtidos na cubagem são analisados empregando-se técnicas de regressão linear e não-linear com o intuito de definir uma equação adequada à predição do volume em árvores não-cubadas. Dependendo do objetivo a que se propõe o uso do tronco, podem ser utilizados métodos de taper, de razão de volumes ou de único volume, cabendo citar os métodos relatados por: Spurr (1952), Campos e Leite (2002), Scolforo (2005), dentre outros.

$\mathrm{Na}$ definição da equação de regressão mais adequada, em geral, são utilizados dados obtidos em árvores-amostra abatidas, o que acarreta dispêndios de recursos, às vezes, altíssimos, porque a cubagem deve ser realizada constantemente nos vários estratos que compõem as populações amostradas. Assim, o alto custo dessa atividade aliado ao tempo, deslocamento das equipes e porte das árvores fazem com que a cubagem seja, muitas vezes, negligenciada na empresa florestal, impactando negativamente as predições volumétricas.

1. Engenheiro Florestal, Doutorando pelo Programa de Pós-Graduação em Engenharia Florestal, Universidade Federal de Lavras, Caixa Postal 37, CEP 37200-000, Lavras (MG). Bolsista CAPES. vclandrade@mailcity.com

2. Engenheiro Florestal, PhD., Professor do Departamento de Ciências Florestais, Universidade Federal de Lavras, Caixa Postal 37, CEP 37200-000, Lavras (MG). calegari@ufla.br

3. Engenheiro Florestal, Dr., Professor do Departamento de Ciências Florestais, Universidade Federal de Lavras, Caixa Postal 37, CEP 37200-000, Lavras (MG). scolforo@ufla.br

Recebido para publicação em 29/11/2002 e aceito em 25/07/2006. 
Em contraposição a esse fato e visando caracterizar o perfil do tronco sem necessitar abater árvores para cubagem, Andrade e Leite (2001) desenvolveram o método da altura relativa tendo por base teórica o seguinte postulado: "Existe um determinado ponto entre 1,3 $\mathrm{m}$ do terreno e a altura total de uma árvore, em que esta ao ser dividida em dois intervalos, possibilita minimizar os erros na descrição do seu taper, pois se considera que o perfil do tronco de uma árvore seja o resultado da interseção de várias retas com coeficientes angulares em intervalos aproximadamente iguais".

Como resultado disso, para se desenvolver o método da altura relativa, os autores recomendaram medir apenas a altura total $(h)$ e os diâmetros situados a 1,3 metros do terreno $\left(d_{1,3}\right)$ e em $h r=h-0,545673 d_{1,3}$, denominado de altura relativa $(h r)$. Em um outro estudo desse método, Andrade (2001) demonstrou ser mais adequado desenvolvê-lo partindo da medição dos diâmetros situados a $0,3 \mathrm{~m}$ (toco), 1,3 m, na altura total $h$ e na altura relativa $h r$, obtida por $h r=(h-2) / 2$.

$\mathrm{Na}$ realidade, ao se utilizar o método altura da relativa, tem-se um cenário em que não é necessário abater árvores para a cubagem. Além desse fato, como identificado em Leite e Andrade (2002), as medições prescritas podem ser feitas em apenas algumas árvores de cada parcela do inventário, que seriam as mesmas árvores amostradas para compor a relação hipsométrica. Essa ação propicia caracterizar o taper das árvores de cada parcela e em cada época de medição, fato equivalente ao de se estar realizando a cubagem com o abate de árvores vinculado a cada uma das parcelas.

O que se procura com este estudo é identificar novas opções para se retratar o tronco das árvores de forma a propiciar maior acurácia ao método da altura relativa, tendo os seguintes objetivos:

a) Identificar novas opções para obter os coeficientes angulares da reta, incluindo a identificação da melhor forma de transformação das variáveis utilizadas;

b) Comparar os coeficientes angulares da reta avaliados neste estudo com o método original;

c) Verificar se o método da altura relativa deve ser desenvolvido empregando todas as árvores que compõem as parcelas de um inventário florestal.

\section{MATERIAL E MÉTODOS}

Este estudo foi realizado utilizando dados de sessenta árvores de eucalipto distribuídas com igual número de indivíduos por classe de $d_{1,3}$ e tendo medições de diâmetros do tronco nas posições: $0,1 \mathrm{~m}, 0,3 \mathrm{~m}$, $0,7 \mathrm{~m}, 1,3 \mathrm{~m}, 2 \mathrm{~m}, 3 \mathrm{~m}, 4 \mathrm{~m}, 5 \mathrm{~m}$, até 1 a $2 \mathrm{~cm}$ de diâmetro com casca.

Utilizando somente as informações de $d_{1,3}, h$ e dos diâmetros localizados em $h_{0,0}(0,3 \mathrm{~m})$ e em $h r=(h-2) / 2$ das sessenta árvores, conforme Andrade (2001), desenvolveu-se o método da altura relativa em sua formulação original de $C A R_{k}$ aplicando-se:

$$
\begin{aligned}
& \text { CAR }_{I}=\frac{1,3-h_{0,0}}{\left(d_{1,3}-d_{h_{0,0}}\right) / 2} \\
& \text { CAR }_{I I}=\frac{1,3-h r}{\left(d_{1,3}-d_{h r}\right) / 2} \\
& \text { CAR }_{I I I}=\frac{h r-h}{\left(d_{h r}\right) / 2}
\end{aligned}
$$

$\mathrm{O}$ que gerou as seguintes equações de taper:

$$
\begin{aligned}
& d_{\left(0,0 \leq h_{i} \leq 1,3\right)}=\frac{2 h_{i}-2,6}{C A R_{I}}+d_{1,3} \\
& d_{\left(1,3<h_{i} \leq h r_{1}\right)}=\frac{2 \cdot h_{i}-2,6}{C A R_{I I}}+d_{1,3} \\
& d_{\left(h r_{1}<h_{i} \leq h r_{3}\right)}=\left(\frac{h_{i}-h r_{1}}{h r_{1}-h r_{2}}\right)\left(\frac{2 h r_{1}-h r_{2}-1,3}{2 C A R_{I I}}+\frac{h-h r_{2}}{2 C A R_{I I I}}+\frac{d_{1,3}}{4}\right)+\frac{h_{i}+h r_{1}-2,6}{C A R_{I I}}+d_{1,3}
\end{aligned}
$$


$d_{\left(h r_{3}<h_{i} \leq h\right)}=\left(\frac{h_{i}-h}{h r_{3}-h}\right)\left[\left(\frac{h r_{3}-h r_{1}}{h r_{1}-h r_{2}}\right)\left(\frac{2 h r_{1}-h r_{2}-1,3}{2 C A R_{I I}}+\frac{h-h r_{2}}{2 C A R_{I I I}}+\frac{d_{1,3}}{4}\right)+\frac{h r_{3}+h r_{1}-2,6}{C A R_{I I}}+d_{1,3}\right]$

Em que: $C A R_{k}=$ coeficiente angular real da reta que passa pelo $k$-ésimo intervalo I, II ou III; $d_{\left(0,0 \leq h_{i} \leq h\right)}=$ diâmetro na $h_{i \text {-ésima }}$ altura desejada entre $0,0 \mathrm{~m} \mathrm{e} \mathrm{h.}$

Nas equações 4, 5, 6 e 7, são utilizados valores reais de $C A R_{k}$, se estes forem obtidos por meio das fórmulas 1, 2 e 3 aplicadas em todas as árvores amostradas pelo inventário. No entanto, no caso da parcela do inventário florestal, quando se tem apenas dados de $d_{1,3} \mathrm{e} h$ das árvores, pode-se obter $C A R_{k}$ empregandose análise de regressão a valores reais de $C A R_{k}$ obtidos para somente algumas árvores.

Utilizar análise de regressão, para obter $C A R_{k}$, pode não proporcionar resultados satisfatórios, porque, em conseqüência baixa correlação de $C A R_{k} \operatorname{com} d_{1,3}$ e $h$, nem sempre se obtém boas estimativas de equações de regressão e, conseqüentemente, boas predições do perfil do tronco.

Uma opção para viabilizar essa metodologia pode ser a transformação de $C A R_{k}$ em $d_{1,3}{ }^{2} h / C A R_{k}$, ou transformar as variáveis envolvidas nas fórmulas 1,2 e 3 fazendo: $d_{h_{0,0}} / d_{1,3}, d_{1,3} / d_{1,3}, d_{h r} / d_{1,3}, h_{0,0} / h$, $1,3 / h, h r / h$ e $h / h$, o que permitiu desenvolver as seguintes fórmulas:

\section{Formulação $C A R_{k} a$}

$$
\begin{aligned}
& \operatorname{CAR}_{I} a=\left(\frac{1,3}{h}-\frac{h_{0,0}}{h}\right) /\left[\frac{\left(d_{1,3} / 2\right)}{\left(d_{1,3} / 2\right)}-\frac{\left(d_{h_{0,0}} / 2\right)}{\left(d_{1,3} / 2\right)}\right]=\left(\frac{1,3-h_{0,0}}{h}\right) /\left(1-\frac{d_{h_{0,0}}}{d_{1,3}}\right) \\
& \text { CAR }_{I I} a=\left(\frac{1,3}{h}-\frac{h r}{h}\right) /\left[\frac{\left(d_{1,3} / 2\right)}{\left(d_{1,3} / 2\right)}-\frac{\left(d_{h r} / 2\right)}{\left(d_{1,3} / 2\right)}\right]=\left(\frac{1,3-h r}{h}\right) /\left(1-\frac{d_{h r}}{d_{1,3}}\right) \\
& \text { CAR }_{I I I} a=\left(\frac{h r}{h}-\frac{h}{h}\right) /\left[\frac{\left(d_{h r} / 2\right)}{\left(d_{1,3} / 2\right)}\right]=\left(\frac{h r}{h}-1\right) /\left(\frac{d_{h r}}{d_{1,3}}\right)
\end{aligned}
$$

\section{Formulação $C A R_{k} b$}

$$
\begin{aligned}
& C A R_{I} b=\left(1,3-h_{0,0}\right) /\left[\frac{\left(d_{1,3} / 2\right)}{\left(d_{1,3} / 2\right)}-\frac{\left(d_{h_{0,0}} / 2\right)}{\left(d_{1,3} / 2\right)}\right]=\left(1,3-h_{0,0}\right) /\left(1-\frac{d_{h_{0,0}}}{d_{1,3}}\right) \\
& C A R_{I I} b=(1,3-h r) /\left[\frac{\left(d_{1,3} / 2\right)}{\left(d_{1,3} / 2\right)}-\frac{\left(d_{h r} / 2\right)}{\left(d_{1,3} / 2\right)}\right]=(1,3-h r) /\left(1-\frac{d_{h r}}{d_{1,3}}\right) \\
& C A R_{I I I} b=(h r-h) /\left[\frac{\left(d_{h r} / 2\right)}{\left(d_{1,3} / 2\right)}\right]=(h r-h) /\left(\frac{d_{h r}}{d_{1,3}}\right)
\end{aligned}
$$

\section{Formulação $C A R_{k} c$}

$$
\begin{aligned}
& C A R_{I} c=\left(\frac{1,3}{h}-\frac{h_{0,0}}{h}\right) /\left(\frac{d_{1,3}}{2}-\frac{d_{h_{0,0}}}{2}\right)=\left(\frac{1,3-h_{0,0}}{h}\right) /\left(\frac{d_{1,3}-d_{h_{0,0}}}{2}\right) \\
& C A R_{I I} c=\left(\frac{1,3}{h}-\frac{h r}{h}\right) /\left(\frac{d_{1,3}}{2}-\frac{d_{h r}}{2}\right)=\left(\frac{1,3-h r}{h}\right) /\left(\frac{d_{1,3}-d_{h r}}{2}\right) \\
& C A R_{I I I} c=\left(\frac{h r}{h}-\frac{h}{h}\right) /\left(\frac{d_{h r}}{2}\right)=\left(\frac{h r}{h}-1\right) /\left(\frac{d_{h r}}{2}\right)
\end{aligned}
$$

Utilizando as fórmulas 8 a 16, desenvolveram-se, algebricamente, as equações para descrever o perfil do tronco segmentado em quatro partes, como se segue. 


\section{Formulação $C A R_{k} a$}

$$
\begin{aligned}
& d_{\left(0,0 \leq h_{i} \leq 1,3\right)}=d_{1,3}\left(1-\frac{1,3-h_{i}}{h C A R_{I} a}\right) \\
& d_{\left(1,3<h_{i} \leq h r_{1}\right)}=d_{1,3}\left(1-\frac{1,3-h_{i}}{h C A R_{I I} a}\right) \\
& d_{\left(h r_{1}<h_{i} \leq h r_{3}\right)}=\left(\frac{h_{i}-h r_{1}}{h r_{1}-h r_{2}}\right)\left(\frac{d_{1,3}}{h}\right)\left(\frac{2 h r_{1}-h r_{2}-1,3}{4 C A R_{I I} a}+\frac{h-h r_{2}}{4 C A R_{I I I} a}+\frac{h}{4}\right)+\left(\frac{d_{1,3}}{h}\right)\left(\frac{h_{i}+h r_{1}-2,6}{2 C A R_{I I} a}+h\right) \\
& d_{\left(h r_{3}<h_{i} \leq h\right)}=\left(\frac{h_{i}-h}{h r_{3}-h}\right)\left[\left(\frac{h r_{3}-h r_{1}}{h r_{1}-h r_{2}}\right)\left(\frac{d_{1,3}}{h}\right)\left(\frac{2 h r_{1}-h r_{2}-1,3}{4 C A R_{I I} a}+\frac{h-h r_{2}}{4 C A R_{I I I} a}+\frac{h}{4}\right)+\left(\frac{d_{1,3}}{h}\right)\left(\frac{h r_{3}+h r_{1}-2,6}{2 C A R_{I I} a}+h\right)\right]
\end{aligned}
$$

\section{Formulação $C A R_{k} b$}

$$
\begin{aligned}
& d_{\left(0,0 \leq h_{i} \leq 1,3\right)}=d_{1,3}\left(1-\frac{1,3-h_{i}}{C A R_{I} b}\right) \\
& d_{\left(1,3<h_{i} \leq h r_{1}\right)}=d_{1,3}\left(1-\frac{1,3-h_{i}}{C A R_{I I} b}\right) \\
& d_{\left(h r_{1}<h_{i} \leq h r_{3}\right)}=\left(\frac{h_{i}-h r_{1}}{h r_{1}-h r_{2}}\right)\left(\frac{d_{1,3}}{4}\right)\left(\frac{2 h r_{1}-h r_{2}-1,3}{C A R_{I I} b}+\frac{h-h r_{2}}{C A R_{I I I} b}+1\right)+\left(\frac{d_{1,3}}{2}\right)\left(\frac{h r_{1}+h_{i}-2,6}{C A R_{I I} b}+1\right) \\
& d_{\left(h r_{3}<h_{i} \leq h\right)}=\left(\frac{h_{i}-h}{h r_{3}-h}\right)\left[\left(\frac{h r_{3}-h r_{1}}{h r_{1}-h r_{2}}\right)\left(\frac{d_{1,3}}{4}\right)\left(\frac{2 h r_{1}-h r_{2}-1,3}{C A R_{I I} b}+\frac{h-h r_{2}}{C A R_{I I I} b}+1\right)+\left(\frac{d_{1,3}}{2}\right)\left(\frac{h r_{3}+h r_{1}-2,6}{C A R_{I I} b}+1\right)\right]
\end{aligned}
$$

Formulação $C A R_{k} c$

$$
\begin{aligned}
& d_{\left(0,0 \leq h_{i} \leq 1,3\right)}=\frac{2 h_{i}-2,6}{h C A R_{I} c}+d_{1,3} \\
& d_{\left(1,3<h_{i} \leq h r_{1}\right)}=\frac{2 h_{i}-2,6}{h C A R_{I I} c}+d_{1,3} \\
& d_{\left(h r_{1}<h_{i} \leq h r_{3}\right)}=\left(\frac{h_{i}-h r_{1}}{h r_{1}-h r_{2}}\right)\left[\left(\frac{1}{h}\right)\left(\frac{2 h r_{1}-h r_{2}-1,3}{2 C A R_{I I} c}+\frac{h-h r_{2}}{2 C A R_{I I I} c}\right)+\frac{d_{1,3}}{4}\right]+\frac{h_{i}+h r_{1}-2,6}{h C A R_{I I} c}+d_{1,3} \\
& d_{\left(h r_{3}<h_{i} \leq h\right)}=\left(\frac{h_{i}-h}{h r_{3}-h}\right)\left\{\left(\frac{h r_{3}-h r_{1}}{h r_{1}-h r_{2}}\right)\left[\left(\frac{1}{h}\right)\left(\frac{2 h r_{1}-h r_{2}-1,3}{2 C A R_{I I} c}+\frac{h-h r_{2}}{2 C A R_{I I I} c}\right)+\frac{d_{1,3}}{4}\right]+\frac{h r_{3}+h r_{1}-2,6}{h C A R_{I I} c}+d_{1,3}\right\}
\end{aligned}
$$

As equações 4 a 7 e 17 a 28 ; geradas pelas fórmulas de $C A R_{k}$ e $C A R_{k} i$, sendo $k=I, I I$ e $I I I$ e $i=a, b$ e $c$, foram utilizadas para fazer predições de diâmetros ao longo do tronco e de volumes comerciais até os diâmetros de $0 \mathrm{~cm}$ (volume total-Vt), $4 \mathrm{~cm}(V 4)$ e de $6 \mathrm{~cm}(V 6)$ com casca. Nesse caso, para obter os volumes comerciais, aplicou-se a fórmula de Smalian em alturas abaixo que $2 \mathrm{~m}$ e, partindo desta, supondo toras com $2 \mathrm{~m}$ de comprimento, aplicou-se a fórmula de Newton até o diâmetro comercial desejado. Para obter o volume total $V t$, somou-se ao volume obtido até o diâmetro de, aproximadamente, $2 \mathrm{~cm}(h c)$ o volume de um cone compreendendo o intervalo entre $h c$ e $h$.

Adicionalmente, considerando que, em árvores em que se tem somente valores de $d_{1,3}$ e $h$, o uso das equações 4 a 7 e 17 a 28 exige o emprego de equações para se obterem os coeficientes angulares $C A R_{k} \mathrm{e}$ $C A R_{K} i$, pois não se tem $d_{h_{0,0}}$ e $d_{h r}$ para gerar valores reais destes. Assim, procedeu-se à simulação desse cenário avaliando-se um grande número de equações de regressão compatíveis, ou seja, que além de fazer boa predição de $C A R_{k}$, também proporcionassem uma boa predição do diâmetro do tronco, uma vez que equações adequadas de $C A R_{k}$ podem fornecer predição de diâmetros negativos para algumas posições no tronco.

Para a seleção da melhor equação de regressão e também avaliação de diâmetros do tronco e dos 
volumes $V t, V 4$ e $V 6$, realizou-se a análise de resíduos por meio de gráficos e das estatísticas:

$$
\begin{aligned}
& D M P=\left[\left(\frac{1}{n}\right) \sum_{i=1}^{n}\left(\frac{Y_{i}-Y_{i}}{Y_{i}}\right)\right] 100, s_{Y_{i} \hat{Y}_{i}}= \pm \frac{\sqrt{\sum_{i=1}^{n}\left(Y_{i}-\hat{Y_{i}}\right)^{2} /(n-2)}}{\bar{Y}} \\
& P= \pm \sqrt{\left(\frac{1,96^{2}}{\chi_{n}^{2}}\right) \sum_{i=1}^{n}\left(\frac{Y_{i}-\hat{Y}_{i}}{Y_{i}}\right)^{2}} .
\end{aligned}
$$

Em que: $D M P=$ desvio médio percentual; $s_{Y \hat{Y}}=$ erro-padrão residual entre valores reais e preditos; $P=$ exatidão obtida pelo teste de qui-quadrado ao nível de 0,05 , conforme Paula Neto et al. (1983); $\hat{Y}_{i}$ e $Y_{i}=$ valor predito e real de $C A R_{k}, C A R_{K} i$, diâmetros do tronco e volumes $V t, V 4$ e $V 6 ; n=$ número de pares de $\hat{Y}_{i}$ e $Y_{i}$; $\bar{Y}=$ valor real médio.

$\mathrm{Na}$ avaliação das equações, procedeu-se à classificação dos resultados obtidos para as estatísticas adotadas. Adotou-se peso 1 para o melhor resultado, peso 2 para o resultado classificado em segundo lugar e assim por diante até a última equação avaliada. O resultado final foi agrupado na média ponderada obtida por: $P M(\%)=\sum($ Peso $*$ Estatística $) / \sum($ Peso $)$.

Uma análise de variância, aplicando o teste conhecido como teste de identidade de Graybill (Regazzi, 1996, Battisti, 2001), foi utilizado para verificar a necessidade de uso de equações para obter $C A R_{k} i$ em cada classe de diâmetro (modelo completo). Para esse propósito, utilizando-se os valores reais de coeficiente angular obtidos por meio da formulação selecionada nas 60 árvores, foi testada a redução provocada na soma de quadrados da regressão por causa do uso de uma única equação para todas as classes de diâmetro (modelo reduzido). Assim, calculou-se a seguinte estatística:

$$
F_{(H o)}=\left[\frac{\operatorname{Re} d u c ̧ \tilde{a} o\left(H_{0}\right)}{(H-1) p}\right]\left[\frac{S Q \operatorname{Re} s(c)}{N-H p}\right]^{-1} .
$$

Em que: Redução $\left(H_{0}\right)=S Q P a r(c)-\operatorname{SQPar}(r) ; \operatorname{SQPar}(c)=$ soma de quadrados de parâmetros do modelo completo; $S Q P a r(r)=$ soma de quadrados de parâmetros do modelo reduzido; $S Q R e s(c)=$ soma de quadrados do resíduo aplicando a equação do modelo completo em cada respectivo estrato; $H=$ número de equações comparadas; $P=$ número de parâmetros do modelo reduzido; $N=$ número total de observações considerando $H$ equações estimadas.

O teste $F_{(H o)}$ foi utilizado para se verificar a existência de igualdade ou diferença no banco de dados envolvendo as classes de diâmetro. Quando $F\left(H_{0}\right)$ resulta em não-significância $\left({ }^{n s}\right)$ ao nível de $5 \%$ de probabilidade, estatisticamente, pode-se inferir que as classes de diâmetro analisadas se constituem em um lote de dados com potencial para estimar a mesma equação de $C A R_{k} i$. Caso contrário, deve-se estimar uma equação de $C A R_{k} i$ a cada classe de diâmetro.

Após selecionada a formulação de $C A R_{K} i$, realizou-se uma comparação com o uso do método da altura relativa tendo $C A R_{k}$ predito pelas equações obtidas por Andrade (2001), que são:

$$
\begin{aligned}
\hat{C A R_{I}} & =\frac{d_{1,3}{ }^{2} h}{\exp \left[0,334654+3,058982 \operatorname{Ln}\left(d_{1,3}\right)\right]} \\
\hat{C A R_{I I}} & =\frac{d_{1,3}{ }^{2} h}{-0,216009 d_{1,3}{ }^{3,220234}}
\end{aligned}
$$


$\hat{C A R}_{I I I}=\frac{d_{1,3}{ }^{2} h}{-0,935519 d_{1,3}{ }^{2,857664}}$

Também, realizou-se inferências sobre a medição dos diâmetros situados a $0,3 \mathrm{~m}, 1,3 \mathrm{~m}$ e em $h r=(h-2) / 2$ adaptando-se os dados das sessenta árvores na simulação de suas medições em todas as árvores. Diante disso, foram definidos os seguintes métodos:

Método I - uso da formulação selecionada de $C A R_{k} i$ nas suas respectivas equações de taper;

Método II - uso das equações 29, 30 e 31 obtidas por Andrade (2001) para fazer a predição de $C A R_{k}$ nas equações de taper 4, 5, 6 e 7;

Método III - uso de valores reais dos coeficientes angulares obtidos pelas fórmulas 1,2 e 3 nas equações de taper 4, 5, 6 e 7 .

É importante ressaltar que o uso de valores reais de coeficientes angulares gera o mesmo valor de diâmetros do tronco independentemente da formulação utilizada, seja de $C A R_{k}$ ou de $C A R_{K} i$. Com isso, o método III poderia se referir a qualquer uma das formulações apresentadas neste estudo, sendo preferido o uso original do método da altura relativa por ser mais fácil de trabalhar com suas respectivas equações de taper, representadas pelas fórmulas 1,2 e 3 aplicadas nas equações 4, 5, 6 e 7 .

Um exemplo do método da altura relativa utilizando o método III é apresentado no apêndice deste estudo, similar ao encontrado em Scolforo (2005) e também todas as transformações algébricas feitas nas fórmulas de coeficientes angulares gerando equações de taper.

\section{RESULTADOS E DISCUSSÃO}

O resultado das análises feitas para se obter o coeficiente angular da reta, que passa em cada intervalo formado no tronco das 60 árvores, intervalos correspondendo à $0,3 \mathrm{~m}$ com 1,3 $\mathrm{m}$ (Intervalo I), $1,3 \mathrm{~m}$ com $h r=(h-2) / 2$ (Intervalo II) e $h r$ com $h$ (Intervalo III), foi a seleção das fórmulas representadas por $C A R_{k} b$, fórmulas 11,12 e 13 .

Das várias equações avaliadas, quanto às predições de $C A R_{k} b$ e diâmetros no tronco, decidiu-se por aquelas apresentadas na Tabela 1 que foram estimadas por meio do modelo linear clássico. Tais equações, conforme indica a não-significância do quadrado médio da redução da regressão $\left(Q M \operatorname{Re} d_{\left(H_{0}\right)}{ }^{\text {ns }}\right)$, foram estimadas tendo os dados agrupados em um único lote em vez de separadamente por classe de diâmetro.

As equações apresentadas na Tabela 1 foram utilizadas para fazer a predição de $C A R_{k} b$ nas equações de taper 21, 22, 23 e 24 definindo-se como método I (MI). Este apresentou melhor acurácia que o método II (MII) e pior que o método III (MIII), conforme se pode ver pela estatística percentual médio (PM) apresentada na Tabela 2 .

Além da Tabela 2 apresentar resultados expressivamente melhores quando se utilizou o método III, tanto para fazer a predição de diâmetros do tronco como para os volumes comerciais $V t, V 4$ e $V 6$, a Figura 1 também indica o referido método como a melhor opção, pois apresentou uma distribuição de resíduos mais uniforme tendo a maioria deles oscilando em torno de $\pm 1 \mathrm{~cm}$.

$\mathrm{Na}$ Figura 2, são apresentados os resíduos obtidos para o volume comercial empregando-se os métodos I, II e III. Observa-se melhor distribuição dos resíduos para os volumes comerciais obtidos pelo método III, conforme já evidenciado anteriormente pelas estatísticas apresentadas na Tabela 2. 
TABELA 1: Estatísticas obtidas para predizer $C A R_{k} b$ nas sessenta árvores simulando ter disponível somente $d_{1,3}$ e $h$; em que: $\hat{\beta}_{i}=$ parâmetros de regressão estimados, $\varepsilon$ =erro aleatório da equação, $X=\left[d_{1,3}(0,5 h-1)\right], L n=$ logarítmo neperiano, $\bar{R}^{2}=$ coeficiente de determinação ajustado, $n s=$ não significativo ao nível de $5 \%$ de probabilidade e $Q M \operatorname{Re} d_{\left(H_{0}\right)}=$ quadrado médio da redução obtida pelo uso dos dados agrupados ao invés de se utilizar uma equação de $C A R_{k} b$ para cada classe de diâmetro.

TABLE 1: Statistics to estimate $C A R_{k} b$ in 60 trees considering to have only $d_{1,3}$ and $h$; available where: $\hat{\beta}_{i}=$ estimated regression parameter, $\varepsilon=$ random error, $X=\left[d_{1,3}(0,5 h-1)\right], L n=$ neperian logaritm, $\bar{R}^{2}=$ coefficient of determination adjusted, $n s=$ non significant to $5 \%$ of probability and $Q M \operatorname{Re} d_{\left(H_{0}\right)}=$ mean squared of the reduction obtained by using grouped data instead the $C A R_{k} b$ equation by diameter class.

\begin{tabular}{l|c|c|c|c|c|c}
\hline Equação & $\hat{\beta_{0}}$ & $\hat{\beta}_{1}$ & $\hat{\beta_{2}}$ & $\hat{\beta_{3}}$ & $\bar{R}^{2}$ & $Q M \operatorname{Re} d_{\left(H_{0}\right)}$ \\
\hline$\left(C A R_{I} b\right)=\hat{\beta}_{0}+\hat{\beta}_{1}(X)+\hat{\beta}_{2}\left(d_{1,3}\right)+\varepsilon$ & $-7,96654$ & $-0,07957$ & 0,90010 & & 62,76 & $5,49429^{\mathrm{ns}}$ \\
$\operatorname{Ln}\left(C A R_{I I} b\right)=\hat{\beta}_{0}+\hat{\beta}_{1}(X)+\hat{\beta}_{2}\left(1 / d_{1,3}\right)+\hat{\beta}_{3} \operatorname{Ln}(h)+\varepsilon$ & $-1,79737$ & 1,58547 & 4,46034 & $-0,00057$ & 81,24 & $0,01905^{\mathrm{ns}}$ \\
$\operatorname{Ln}\left(C A R_{I I I} b\right)=\hat{\beta}_{0}+\hat{\beta}_{1}(h)+\hat{\beta}_{2}(1 / X)+\varepsilon$ & 2,165118 & 0,03594 & $-13,57630$ & & 97,63 & $0,00148^{\mathrm{ns}}$ \\
\hline
\end{tabular}
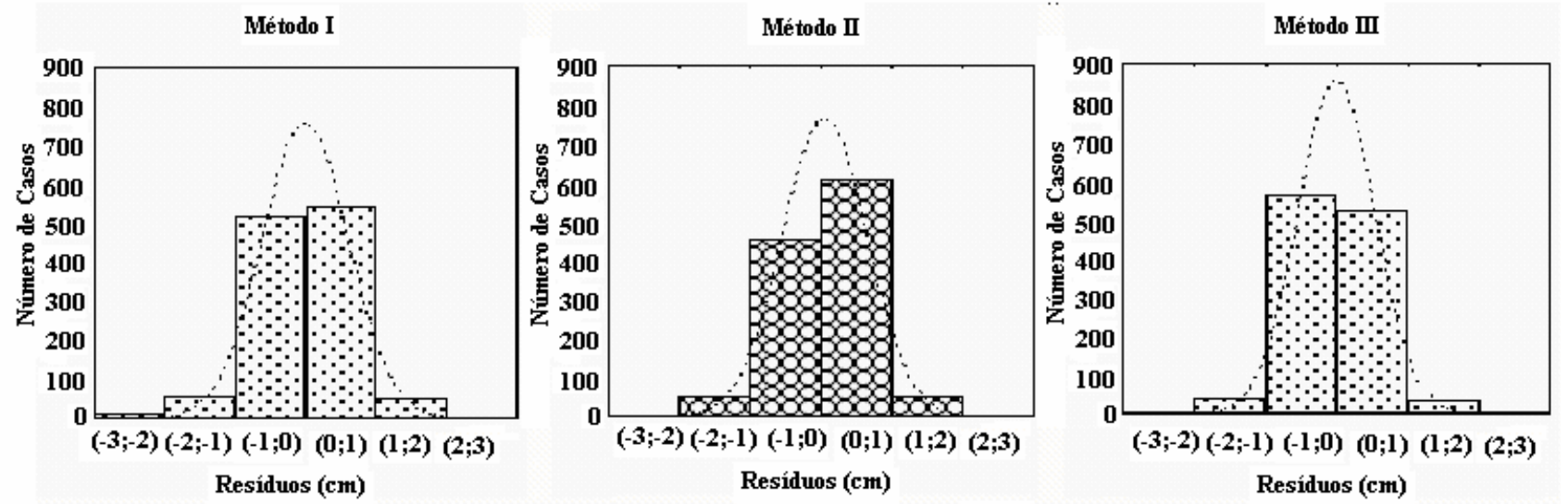

FIGURA 1: Histograma dos resíduos obtidos para os métodos I, II e III. FIGURE 1: Residual histogram for methods I, II, and III.

Diante dos resultados obtidos, evidencia-se que, para caracterizar o perfil do tronco de eucalipto empregando geometria analítica, se deve utilizar coeficientes angulares reais da reta e evitar obtê-los por meio de análise de regressão. Portanto, deve-se medir os diâmetros localizados nas alturas $0,3,1,3$ e $h r=(h$ 2)/2 metros do terreno em todas as árvores amostradas pelo inventário, o que permite inferir sobre o método da altura relativa ser uma importante ferramenta para se alterar drasticamente a estratégia de cubagem rigorosa, sem a necessidade de abater árvores-amostra.

As estatísticas da Tabela 2 indicam, ainda, que, ao se utilizar o método da altura relativa para caracterizar o perfil do tronco, em média, há perdas na acurácia a medida em que a $h_{\text {iésima }}$ altura se distancia em direção de $h$, sobretudo em alturas maiores que $h r_{1}$. Como se tratam de diâmetros menores, os erros evidenciados podem não influenciar indesejavelmente a predição do volume.

Considerando o método III, houve uma diferença entre diâmetros preditos e reais $(P)$ inferior a $\pm 8 \%$ em alturas menores que $h r_{1}$. Essas estatísticas refletiram no volume comercial, indicando uma diferença entre volumes preditos e reais de $\pm 5 \%$ para $V t$ e $V 4$ e de $\pm 12 \%$ para $V 6$, bem abaixo dos resultados obtidos 
para o método I.

TABELA 2: Critérios estatísticos adotados para avaliar os métodos I (MI), II (MII) e III (MIII); em que: $\mathrm{dI}=$ diâmetros em alturas menores que 1,3 metros, $\mathrm{dII}=$ diâmetros entre $1,3 \mathrm{~m}$ e $h r_{1}$, dIII = diâmetros entre $h r_{1}$ e $h r_{3}$, dIV = diâmetros entre $h r_{3}$ e $h c$.

TABLE 2: Statistical criteria adopted to evaluate the methods I (MI), II (MII) e III (MIII), where: $\mathrm{dI}=$ diameter taken in height smaller than 1,3 meters, $\mathrm{dII}=$ diameter between $1,3 \mathrm{~m}$ and $h r_{1}$, $\mathrm{dIII}=$ diameter between $h r_{1}$ and $h r_{3}$, dIV = diameter between $h r_{3}$ and $h c$.

\begin{tabular}{|c|c|c|c|c|c|}
\hline Método & & $D M P(\%)$ & $s_{Y \hat{Y}}(\%)$ & $P(\%)$ & $P M(\%)$ \\
\hline $\mathrm{dI}-\mathrm{MI}$ & & $-0,60$ & 5,09 & 8,60 & 4,37 \\
\hline dII - MI & & 1,58 & 3,80 & 8,73 & 4,70 \\
\hline dIII - MI & & $-2,75$ & 7,98 & 16,60 & 7,38 \\
\hline dIV - MI & & $-3,58$ & 13,96 & 29,22 & 13,20 \\
\hline Total & & & & & 29,64 \\
\hline$\overline{\mathrm{dI}-\mathrm{MII}}$ & & $-0,50$ & 4,67 & 8,29 & 4,15 \\
\hline dII - MII & & 2,05 & 4,01 & 9,38 & 5,14 \\
\hline dIII - MII & & $-0,45$ & 7,71 & 18,85 & 11,92 \\
\hline dIV - MII & & $-0,65$ & 15,23 & 28,96 & 14,80 \\
\hline Total & & & & & 36,02 \\
\hline dI - MIII & & $-0,16$ & 3,99 & 6,83 & 3,55 \\
\hline dII - MIII & & 1,40 & 3,18 & 7,01 & 3,86 \\
\hline dIII - MIII & & $-3,42$ & 7,12 & 16,34 & 2,64 \\
\hline dIV - MIII & & $-4,70$ & 13,86 & 30,40 & 12,99 \\
\hline Total & & & & & 23,05 \\
\hline$V t-\mathrm{MI}$ & & 0,81 & 5,87 & 9,56 & 5,48 \\
\hline V4 - MI & & 0,37 & 5,88 & 11,34 & 5,87 \\
\hline$V 6-\mathrm{MI}$ & & 0,07 & 6,51 & 18,48 & 8,09 \\
\hline Total & & & & & 19,44 \\
\hline$V t-\mathrm{MII}$ & & 2,80 & 5,39 & 11,46 & 6,69 \\
\hline V4 - MII & & 2,22 & 5,50 & 12,94 & 7,06 \\
\hline V6 - MII & & 1,70 & 5,78 & 19,64 & 9,45 \\
\hline Total & & & & & 23,20 \\
\hline$V t-$ MIII & & 0,52 & 2,64 & 5,47 & 2,88 \\
\hline V4 - MIII & & 0,92 & 2,86 & 5,36 & 3,05 \\
\hline V6 - MIII & & 0,38 & 3,43 & 12,32 & 5,38 \\
\hline Total & & & & & 11,30 \\
\hline \multirow[t]{3}{*}{ Geral: } & MI & $49,08 \%$ & & & \\
\hline & MII & $59,21 \%$ & & & \\
\hline & MIII & $34,35 \%$ & & & \\
\hline
\end{tabular}



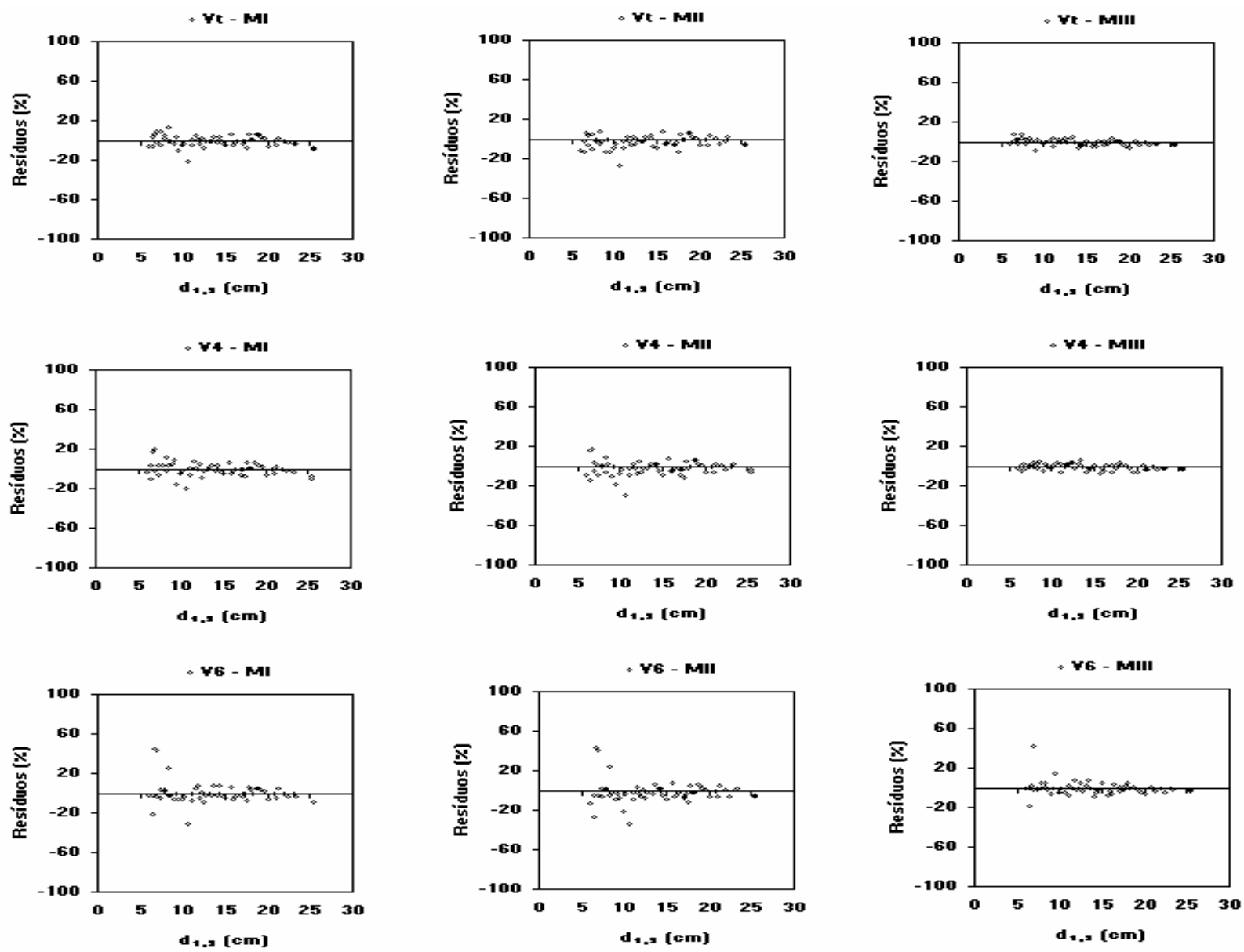

FIGURA 2: Resíduos percentuais em relação ao $d_{1,3}$ obtidos para os volumes comerciais $V t, V 4$ e $V 6$ empregando-se os métodos I, II e III.

FIGURE 2: Residual in percentage based on $d_{1,3}$ obtained for the merchantable volumes $V t, V 4$ and $V 6$ by using the methods I, II, and III.

\section{CONCLUSÕES}

Os resultados obtidos neste estudo permitiram concluir que:

O método da altura relativa faz predição do volume com perdas na acurácia à medida que se aumenta o diâmetro comercial, ocorrendo o inverso para estimativas de diâmetros do tronco;

O método da altura relativa deve considerar as fórmulas 11, 12 e 13 quando for utilizada análise de regressão para obter $C A R_{k} b$ nas equações de perfil do tronco 21, 22, 23 e 24;

Não se deve usar equações de regressão tendo a forma funcional: $\left(d_{1,3}{ }^{2} h / C A R_{k}\right)=f\left(d_{1,3}, h\right)$ para obter o coeficiente angular nas equações de perfil do tronco geradas por geometria analítica;

Em inventários florestais ou em atividades de pesquisa, nas quais se espera melhor acurácia do método da altura relativa, deve-se mensurar $d_{h_{0,0}}, d_{1,3} \mathrm{e} d_{h r}$ em todas as árvores que delimitam as parcelas.

\section{REFERÊNCIA BIBLIOGRÁFICA}

ANDRADE, V.C.L.; LEITE, H.G. Uso da geometria analítica para descrever o taper e quantificar o volume de árvores individuais. Revista Árvore, v. 25, n. 4, p. 481-486, 2001.

ANDRADE, V. C. L. Um método para descrever o perfil do tronco em árvores de eucalipto utilizando geometria analítica. Viçosa, 2001. 74f. Dissertação (Mestrado em Ciência Florestal) - Universidade Federal de Viçosa, Viçosa, 2001.

BATTISTI, I.D.E. Comparação entre modelos de regressão com uma aplicação em Biometria Florestal. Lavras, 
2001. 86f. Dissertação (Mestrado em Estatística e Experimentação Agropecuária) - Universidade Federal de Lavras, Lavras, 2001.

CAMPOS, J.C.C; LEITE, H.G. Mensuração florestal: perguntas e respostas. Viçosa: UFV, 2002. 407p.

FINGER, C.A.G. Fundamentos de biometria florestal. Santa Maria: UFSM/CEPEF/FATEC, 1992. 270p.

LEITE, H.G.; ANDRADE, V.C.L. Um método para condução de inventários florestais sem o uso de equações volumétricas. Revista Árvore, v.26, n.3, p.321-328, 2002.

MACHADO, S.A., FIGUEIREDO-FILHO, A. Dendrometria. Curitiba: A. Figueiredo Filho, 2003. 309p.

PAULA NETO, F.; SOUZA, A.L..; et al. Análise de equções volumétricas para Eucalyptus spp., segundo o método de regeneração na região de José de Melo. Revista Árvore, v. 7, n. 1, p. 56-70, 1983.

REGAZZI, A.J. Teste para verificar a identidade de modelos de regressão. Pesquisa Agropecuária Brasileira, v. 31, n. 1, p. 1-17, 1996.

SCOLFORO, J.R.S. Biometria Florestal: Parte I: modelos de regressão linear e não linear; Parte II: modelos para relação hipsométrica, volume, afilamento e peso de matéria seca. Lavras: UFLA/ FAEPE. 2005. 352p.

SPURR, P.R. Forest Inventory. New York: Ronald Press Company, 1953, 476p. 
A P E E D I C E 


\section{DEDUÇÕES DAS EQUAÇÕES DE TAPER UTILIZANDO O MÉTODO III}

Neste item, seguindo-se a seqüência apresentada em Andrade (2001), algebricamente, são deduzidas as equações de perfil do tronco 4, 5, 6 e 7 partindo das fórmulas 1, 2 e 3 utilizadas no método III. Para os demais métodos, segue-se a mesma seqüência.

Dos valores necessários ao desenvolvimento do método da altura relativa, com a $j$-ésima árvore em pé, é preciso apenas medir o diâmetro situado a $0,3\left(h_{0,0 \mathrm{j}}\right), 1,3 \mathrm{e}$ em $h r_{j}$ metros do terreno dividindo-a nos intervalos I, II e III. Porém, para se utilizar as equações de taper obtidas, a árvore é dividida em quatro novos segmentos, sendo $h_{0,0 j} \operatorname{com} 1,3 \mathrm{~m}, 1,3 \mathrm{~m} \operatorname{com} h r_{1 j}, h r_{1 j} \operatorname{com} h r_{3 j}$ e $h r_{3 j} \operatorname{com} h_{j}$.

Conforme Andrade (2001), partindo das fórmulas:

$\left(C A R_{I}\right)_{j}=\left[2,6-2\left(h_{0,0}\right)_{j}\right] /\left(d_{1,3}-d_{h_{0,0}}\right)_{j}$

$\left(C A R_{I I}\right)_{j}=\left[2,6-2 h r_{j}\right] /\left(d_{1,3}-d_{h r}\right)_{j}$

$\left(C A R_{I I I}\right)_{j}=\left(2 h r_{j}-2 h_{j}\right) /\left(d_{h r}\right)_{j}$

Respectivamente, obtem-se que:

$\left(d_{I}\right)_{j}=\left[2\left(h_{i}\right)_{j}-2,6\right] /\left(C A R_{I}\right)_{j}+d_{1,3}$

$\left(d_{I I}\right)_{j}=\left[2\left(h_{i}\right)_{j}-2,6\right] /\left(C A R_{I I}\right)_{j}+d_{1,3 j}$

$\left(d_{I I I}\right)_{j}=\left[2\left(h_{i}\right)_{j}-2 h_{j}\right] /\left(C A R_{I I I}\right)_{j}$

Sendo $C A R_{k j}$ o coeficiente angular real da reta que passa pelo $k$-ésimo intervalo delimitado na $j$ ésima árvore, com $k=\mathrm{I}$, II e III; $\left(d_{h_{0,0}}\right)_{j}$ o diâmetro medido em $0,3 \mathrm{~m} ;\left(d_{h r}\right)_{j}$ o diâmetro medido em $h r_{j}=\left(h_{j}-2\right) / 2$; e $d_{k j}$ o diâmetro do tronco na $h_{i-\text { ésima }}$ altura desejada.

As equações 32, 33 e 34 são utilizadas de forma a segmentar o tronco das árvores simulando métodos de cubagem. No segmento entre $0,0 \mathrm{~m}$ a $1,3 \mathrm{~m}$, utiliza-se a equação $32 \mathrm{e}$, no segmento entre $1,3 \mathrm{~m}$ a $\left(h r_{1}\right)_{j}=\left(h_{j}-1,7\right) / 1,7$, utiliza-se a equação 33 .

Partindo de 33 e 34, obtem-se os diâmetros em $\left(h r_{1}\right)_{j}$ e $\left(h r_{2}\right)_{j}=\left(h_{j}-1,4\right) / 1,4$ aplicando-se:

$$
\begin{aligned}
& \left(d_{h r_{1}}\right)_{j}=\left[2\left(h r_{1}\right)_{j}-2,6\right] /\left(C A R_{I I}\right)_{j}+d_{1,3} \\
& \left(d_{h r_{2}}\right)_{j}=\left[\left(h r_{2}\right)_{j}-1,3\right] /\left(C A R_{I I}\right)_{j}+\left[\left(h r_{2}\right)_{j}-h_{j}\right] /\left(C A R_{I I I}\right)_{j}+d_{1,3 j} / 2
\end{aligned}
$$

Em seguida, utiliza-se a fórmula: $\left(C A R_{I V}\right)_{j}=\left[2\left(h r_{1}\right)_{j}-2\left(h r_{2}\right)_{j}\right] /\left[\left(d_{h r_{1}}\right)_{j}-\left(d_{h r_{2}}\right)_{j}\right]$, a qual, após transformações algébricas apresentadas em Andrade (2001), resulta na seguinte equação de taper:

$$
\begin{aligned}
\left(d_{I V}\right)_{j}= & {\left[2\left(h_{i}\right)_{j}-2\left(h r_{1}\right)_{j}\right] /\left(C A R_{I V}\right)_{j}+\left(d_{h r_{1}}\right)_{j} } \\
& \text { ou } \\
\left(d_{I V}\right)_{j} & =\left[2\left(h_{i}\right)_{j}-2\left(h r_{1}\right)_{j}\right] /\left(C A R_{I V}\right)_{j}+\left[2\left(h r_{1}\right)_{j}-2,6\right] /\left(C A R_{I I}\right)_{j}+d_{1,3 j}
\end{aligned}
$$

A média entre as equações 33 e 35 resulta na equação de taper que deve ser utilizada no segmento entre $\left(h r_{1}\right)_{j}$ e $\left(h r_{3}\right)_{j}=\left(h_{j}-1,1\right) / 1,1$, cuja forma reduzida é a seguinte:

$$
d_{\left(h r_{1}<h_{i} \leq h h_{3}\right)_{j}}=\left(h r_{1}-h r_{2}\right)_{j}^{-1}\left[\begin{array}{l}
\frac{h r_{1}\left(h r_{2}-2 h r_{1}+2 h_{i}+1,3\right)-h_{i}\left(h r_{2}+1,3\right)}{2 C A R_{I I}} \\
+\frac{\left(h r_{2}-h\right)\left(h r_{1}-h_{i}\right)}{2 C A R_{I I}}+\frac{d_{1,3}\left(h_{i}-h r_{1}\right)}{4}
\end{array}\right]_{j}+\frac{\left(h_{i}+h r_{1}-2,6\right)_{j}}{\left(C A R_{I I}\right)_{j}}+\left(d_{1,3}\right)
$$

Como a equação 36 é utilizada até uma altura $\left(h r_{3}\right)_{j}$, então, partindo desta, calcula-se o diâmetro $\left(d_{h r_{3}}\right)_{j}$ e utiliza-se a fórmula: $\left(C A R_{V}\right)_{j}=\left[2\left(h r_{3}\right)_{j}-2 h_{j}\right] /\left(d h r_{3}\right)_{j}$ gerando a seguinte equação de taper que deve ser utilizada no segmento entre $\left(h r_{3}\right)_{j}$ a $h j$ : 


$$
d_{\left(h h_{3}<h_{i} \leq h\right)_{j}}=\left[\frac{\left(h_{i}-h\right)}{\left(h r_{3}-h\right)}\right]_{j}\left\{\left(h r_{1}-h r_{2}\right)_{j}^{-1}\left[\begin{array}{c}
\frac{h r_{1}\left(h r_{2}-2 h r_{1}+2 h r_{3}+1,3\right)-h r_{3}\left(h r_{2}+1,3\right)}{2 C A R_{I I}} \\
+\frac{\left(h r_{2}-h\right)\left(h r_{1}-h r_{3}\right)}{2 C A R_{I I I}}+\frac{d_{1,3}\left(h r_{3}-h r_{1}\right)}{4}
\end{array}\right]_{j}+\frac{\left(h r_{3}+h r_{1}-2,6\right)_{j}}{\left(C A R_{I I}\right)_{j}}+d_{1,3 j}\right\}
$$

\section{UM EXEMPLO UTILIZANDO O MÉTODO III}

Partindo das medições realizadas numa árvore em pé apresentada na Tabela 3, calcular o seu volume utilizando o método da altura relativa.

O primeiro passo a ser realizado é o cálculo do coeficiente angular real da reta $C A R_{k}$ em cada intervalo formado por $0,3 \mathrm{~m}$ a $1,3 \mathrm{~m}$ (Intervalo I), $1,3 \mathrm{~m}$ a $h r$ (Intervalo II) e $h r$ a $h$ (Intervalo III). Então, conforme as fórmulas 1,2 e 3 , tem-se que:

$$
\begin{aligned}
& C A R_{I}=\frac{1,3-h_{0,0}}{\left(d_{1,3}-d_{h_{0,0}}\right) / 2}=\frac{1,3-0,3}{(14,8-17,0) / 2}=-0,90909, \\
& C A R_{I I}=\frac{1,3-h r}{\left(d_{1,3}-d_{h r}\right) / 2}=\frac{1,3-8}{(14,8-9,7) / 2}=-2,62745 \mathrm{e} \\
& C A R_{I I I}=\frac{h r-h}{d_{h r} / 2}=\frac{8,0-18,0}{9,7 / 2}=-2,06186 .
\end{aligned}
$$

TABELA 3: Diâmetros ao longo do fuste de uma árvore mensurada em pé.

TABLE 3: Diameters across the standing up tree stem.

\begin{tabular}{lc}
\hline Alturas de medição $-h_{i}(\mathrm{~m})$ & Diâmetro a várias alturas $-d_{i}(\mathrm{~cm})$ \\
\hline Altura do toco $=0,3$ & 17,0 \\
Altura do peito $=1,3$ & 14,8 \\
Altura relativa $=8,0$ & 9,7 \\
Altura total $=18,0$ & 0,0 \\
\hline
\end{tabular}

Após realizados os cálculos de $C A R_{k}$, são preditos os diâmetros em diferentes posições ao longo do tronco aplicando-se uma fórmula de cubagem tradicional, obtendo-se o volume da árvore. Neste exemplo, será predito o diâmetro nas posições 0,$1 ; 0,4 ; 0,7 ; 1,0 ; 2,0 ; 3,0 ; 4,0 ; 5,0$; até um diâmetro próximo de $2 \mathrm{~cm}$ $(h c)$.

Antes da predição de diâmetros ao longo do tronco, é necessário ainda o cálculo de três novas alturas relativas, as quais são:

$$
\begin{aligned}
& h r_{1}=\frac{(h-1,7)}{1,7}=\frac{(18-1,7)}{1,7}=9,59, \\
& h r_{2}=\frac{(h-1,4)}{1,4}=\frac{(18-1,4)}{1,4}=11,86 \mathrm{e} \\
& h r_{3}=\frac{(h-1,1)}{1,1}=\frac{(18-1,1)}{1,1}=15,36 .
\end{aligned}
$$

Para $\mathbf{0 , 0} \leq \boldsymbol{h}_{i} \leq \mathbf{1 , 3} \mathbf{m}$ : aplicação da equação 32 fazendo $d_{I}$ igual a $d_{h i}$

$$
\begin{aligned}
& d_{h i}=\frac{2 h_{i}-2,6}{C A R_{I}}+d_{1,3} . \\
& d_{h_{0,1}}=\frac{(2 * 0,1)-2,6}{-0,90909}+14,8=17,44 ; \\
& d_{h_{0,1}}=\frac{(2 * 0,1)-2,6}{-0,90909}+14,8=16,78 ;
\end{aligned}
$$




$$
\begin{aligned}
& d_{h_{0,7}}=\frac{(2 * 0,7)-2,6}{-0,90909}+14,8=16,12 \mathrm{e} \\
& d_{h_{1,0}}=\frac{(2 * 1,0)-2,6}{-0,90909}+14,8=15,46 .
\end{aligned}
$$

Para 1,3 $<\boldsymbol{h}_{\boldsymbol{i}} \leq \boldsymbol{h} \boldsymbol{r}_{1}$ : aplicação da equação 33 fazendo $d_{I I}$ igual a $d_{h i}$

$$
\begin{aligned}
& d_{h i}=\frac{2 h_{i}-2,6}{C A R_{I I}}+d_{1,3} . \\
& d_{h_{2,0}}=\frac{(2 * 2,0)-2,6}{-2,62745}+14,8=14,27 ; \\
& d_{h_{3,0}}=\frac{(2 * 3,0)-2,6}{-2,62745}+14,8=13,51 ; \\
& d_{h_{4,0}}=\frac{(2 * 4,0)-2,6}{-2,62745}+14,8=12,74 ; \\
& d_{h_{5,0}}=\frac{(2 * 5,0)-2,6}{-2,62745}+14,8=11,98 ; \\
& d_{h_{6,0}}=\frac{(2 * 6,0)-2,6}{-2,62745}+14,8=11,22 ; \\
& d_{h_{7,0}}=\frac{(2 * 7,0)-2,6}{-2,62745}+14,8=10,46 \mathrm{e} \\
& d_{h_{9,0}}=\frac{(2 * 9,0)-2,6}{-2,62745}+14,8=8,94 .
\end{aligned}
$$

Para $\boldsymbol{h} \boldsymbol{r}_{\mathbf{1}}<\mathbf{h}_{\mathbf{i}} \leq \boldsymbol{h} \boldsymbol{r}_{3}$ : aplicação da média entre as equações 33 e 35 fazendo $d_{I I}$ e $d_{I V}$ iguais a $d_{h i}$ ou da equação resultante na sua forma reduzida que é a de número 36

$$
\begin{aligned}
& d_{\left(h r_{1}<h_{i} \leq h r_{3}\right)_{j}}=\left(h r_{1}-h r_{2}\right)_{j}^{-1}\left[\begin{array}{l}
\frac{h r_{1}\left(h r_{2}-2 h r_{1}+2 h_{i}+1,3\right)-h_{i}\left(h r_{2}+1,3\right)}{2 C A R_{I I}} \\
+\frac{\left(h r_{2}-h\right)\left(h r_{1}-h_{i}\right)}{2 C A R_{I I}}+\frac{d_{1,3}\left(h_{i}-h r_{1}\right)}{4}
\end{array}\right]_{j}+\frac{\left(h_{i}+h r_{1}-2,6\right)_{j}}{\left(C A R_{I I}\right)_{j}}+\left(d_{1,3 j}\right) . \\
& d_{h_{10}}=(9,59-11,86)^{-1}\left[\frac{\frac{9,59(11,86-2 * 9,59+2 * 10+1,3)-10(11,86+1,3)}{2 *-2,62745}+}{\frac{(11,86-18)(9,59-10)}{2-2,06186}+\frac{14,8(10-9,59)}{4}}\right]+\frac{(10+9,59-2,6)}{2,62745}+14,8=8,14 \text {; } \\
& d_{h_{11}}=(9,59-11,86)^{-1}\left[\frac{\frac{9,59(11,86-2 * 9,59+2 * 11+1,3)-11(11,86+1,3)}{2 *-2,62745}+}{\frac{(11,86-18)(9,59-11)}{2-2,06186}+\frac{14,8(11-9,59)}{4}}\right]+\frac{(11+9,59-2,6)}{2,62745}+14,8=7,29 \text {; } \\
& d_{h_{12}}=(9,59-11,86)^{-1}\left[\frac{\frac{9,59(11,86-2 * 9,59+2 * 12+1,3)-12(11,86+1,3)}{2 *-2,62745}+}{\frac{(11,86-18)(9,59-12)}{2-2,06186}+\frac{14,8(12-9,59)}{4}}\right]+\frac{(12+9,59-2,6)}{2,62745}+14,8=6,44 \text {; } \\
& d_{h_{13}}=(9,59-11,86)^{-1}\left[\frac{\frac{9,59(11,86-2 * 9,59+2 * 13+1,3)-13(11,86+1,3)}{2 *-2,62745}+}{\frac{(11,86-18)(9,59-13)}{2-2,06186}+\frac{14,8(13-9,59)}{4}}\right]+\frac{(13+9,59-2,6)}{2,62745}+14,8=5,59 \text {; }
\end{aligned}
$$




$$
\begin{aligned}
& d_{h_{14}}=(9,59-11,86)^{-1}\left[\frac{\frac{9,59(11,86-2 * 9,59+2 * 14+1,3)-14(11,86+1,3)}{2 *-2,62745}+}{\frac{(11,86-18)(9,59-14)}{2-2,06186}+\frac{14,8(14-9,59)}{4}}\right]+\frac{(14+9,59-2,6)}{2,62745}+14,8=4,74 \mathrm{e} \\
& d_{h_{15}}=(9,59-11,86)^{-1}\left[\frac{\frac{9,59(11,86-2 * 9,59+2 * 15+1,3)-15(11,86+1,3)}{2 *-2,62745}+}{\frac{(11,86-18)(9,59-15)}{2-2,06186}+\frac{14,8(15-9,59)}{4}}\right]+\frac{(15+9,59-2,6)}{2,62745}+14,8=3,89 .
\end{aligned}
$$

Para $\boldsymbol{h} \boldsymbol{r}_{3}<\boldsymbol{h}_{\boldsymbol{i}} \leq \boldsymbol{h}$ : aplicação da equação 37

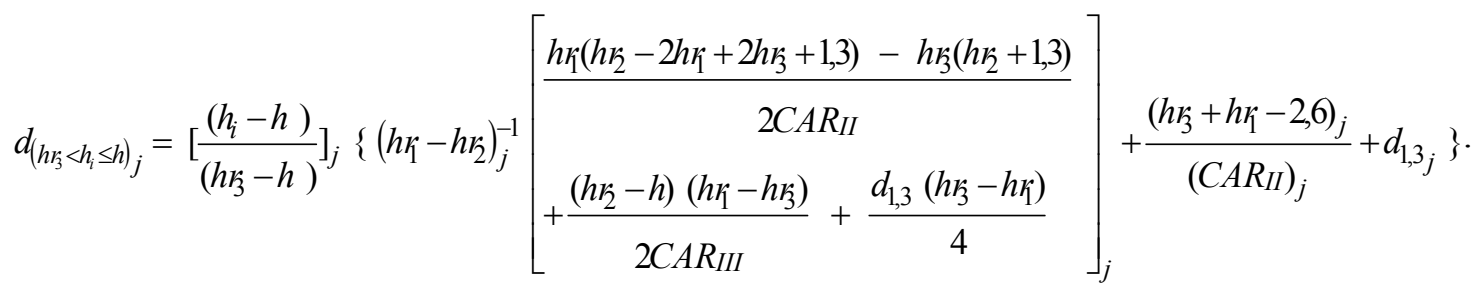

$$
\begin{aligned}
& d_{h_{16}}=\left[\frac{(16-18)}{(15,36-18)}\right]\left\{\left[\frac{\frac{9,59(11,86-2 * 9,59+2 * 15,36+1,3)-15,36(11,86+1,3)}{2 *-2,62745}+}{\frac{(11,86-18)(9,59-15,36)}{2 *-2,06186}+\frac{14,8(15,36-9,59)}{4}}\right]-\frac{(15,36+9,59-2,6)}{-2,62745}+14,8\right\}=2,72
\end{aligned}
$$

Na Tabela 2, podem ser observados todos os diâmetros preditos pelo método da altura relativa, bem como o volume $V_{i}$ utilizando a fórmula de Smalian. Observa-se também que se utilizar o tronco da árvore em toras de 2,0 m de comprimento, o volume comercial $V 6$ é 0,1281 e o $V 4$ é 0,1330 , sendo $V t$ igual a 0,1366 , ambos os volumes $\mathrm{em}^{3}$ com casca.

TABELA 4: Volume com casca calculado pela fórmula de Smalian partindo dos diâmetros com casca preditos pelo método da altura relativa.

\begin{tabular}{|c|c|c|c|c|c|c|c|c|c|c|c|}
\hline $\mathrm{m}$ & 0,1 & 0,4 & 0,7 & 1,0 & 1,3 & 2,0 & 3,0 & 4,0 & 5,0 & 6,0 & 7,0 \\
\hline$d_{i} \mathrm{pr}$ & 17,44 & 16,78 & 16,12 & 15,46 & 14,80 & 14,27 & 13,51 & 12,74 & 11,98 & 11,22 & 10,46 \\
\hline${ }_{i} \mathrm{Sn}$ & 0,0024 & 0,0093 & $\begin{array}{ll}30,0157 \\
\end{array}$ & 0,0215 & $\begin{array}{ll}5 & 0,0269 \\
\end{array}$ & 0,0386 & $\begin{array}{ll}6 & 0,0537 \\
\end{array}$ & $\begin{array}{ll}7 & 0,0673 \\
\end{array}$ & $\begin{array}{ll}3 & 0,0793 \\
\end{array}$ & $\begin{array}{ll}3 & 0,08 \\
\end{array}$ & 0,09 \\
\hline & 8,0 & 9, & 10 & 1 & 12,0 & 13,0 & 4 & 1 & 1 & 18 & \\
\hline & 9,7 & 8,9 & 8, & 7 , & 6,4 & 5, & 4, & 3,8 & 2, & 0 , & \\
\hline$i$ Smaliar & 0,1071 & 0,1139 & 0,1197 & 0,1244 & 0,1281 & 0,1309 & $\begin{array}{ll}9 & 0,1330 \\
\end{array}$ & $\begin{array}{lll}0 & 0,1345 \\
\end{array}$ & $\begin{array}{ll}5 & 0,1354 \\
\end{array}$ & 0,1366 & \\
\hline
\end{tabular}

TABLE 4: Volume inside bark calculated by Smalian formula based on diameter and bark predicted by relative height method. 